\title{
The Effects of Word-of-mouth on the Trend of Movie Box Office Performance: Take Douban Movie's Rating Platform as an Example
}

\author{
Guangpei Chen
}

\author{
School of Management, Northeastern University at Qinhuangdao, Qinhuangdao 066004, China \\ corleonecc@163.com
}

Keywords: Word of mouth, Douban Movie, China's movie market, rating of movies.

\begin{abstract}
During the slowdown in the growth of China's movie market, word of mouth (WOM) seems to exert more influence on the trend of box office performance. The objective of this paper is to measure the impact of WOM (valence and volume) on the trend of movie box office performance, differing from previous studies which research on the total revenues of movies. We extract data from Maoyan Pro and Douban Movie, and investigate the correlation between the trend of box office performance and WOM valence and volume. We conduct our study in the context of China's movie market while past studies are mainly based on foreign markets. We find that there exists a negative relationship between these three variables investigated.
\end{abstract}

\section{Introduction}

Overview. After growing at an average rate of $35 \%$ for 13 consecutive years, China, the second largest movie market in the world, seems to reach a stage saturation with only $3 \%$ rate of growing in the year of 2016. The name and popularity of directors and casts seemingly don't work anymore. By comparison, the quality of movies is becoming increasingly important in determining the box office. As the carriers of quality, critical reviews, specifically the so-called word of mouth (WOM), serve as a tool to immediately disseminate post-purchase quality evaluations provided by consumers who have watched the movies, thus diminishing the information asymmetry that has existed between producers and consumers (Thorsen, Caroline and Fabian 2013) [1]. In fact,more than one-third of Americans actively seek the advice of film critics (The Wall Street Journal 2001), and approximately one out of every three moviegoers says they choose movies because of favorable reviews (Basuroy, Chatterjee and Ravid 2003), giving implications on the transformation of off-line behaviors of consumers, especially in the movie industry where the role of critics may be most prominent (Eliashberg, Shugan 1997; Holbrook 1999; West and Broniarczyk 1998) [2-6]. Consumers no longer follow their favourite idols starring in the movies with no regard to the quality of the movies. Instead, consumers tend to have more rational behaviors and make considerate decisions, taking account of various kinds of considerations, among which the WOM is getting more and more influential.

Although scholars have been arguing over which component of WOM contributes extraordinarily to the box office performance- the volume of reviews (Liu 2006, Duan et. al. 2008) and the valence of reviews (Liu 2006, Duan et. al. 2008, Chevalier and Mayzlin 2006) in the past research, they have reached a consensus that much more attention should be paid to WOM regardless of controversies [7-9].To put it from another way, WOM is playing a significant role in determining the box office, whether it is positive or not. This consensus is underpinned by massive experimental results and empirical data, and it is also reflected in the market performance of movies.

Nevertheless, under the consensus there are still various different points of view towards the precise correlation between box office performance and WOM. While Liu (2006) and Duan et al (2008) find that the volume of user reviews has a positive impact on future box-office revenues of movies, Chintagunta, Gopinath and Venkataraman (2010) find that it is the valence that seems to matter and not the volume $[7-8,10]$. Their findings conflict with previous studies that have found 
that the main driver of box-office performance is the volume of reviews. However, in the middle of these two diametrically opposed opinions, Duan, Gu and Whinston (2008) succeed in establishing a connection between the volume and valence, showing that user ratings do not directly influence box office revenue whereas they affect box-office revenue indirectly by significantly influencing WOM volume and WOM volume in turn leads to higher box office performance [8]. In addition to these two mainstream points of view, there are other opinions which help to constitute the theoretical network of impact factors of movies' market performance. Wasserman, Mukherjee, Scott, Zeng and Radicchi (2015) argue that to make a film more notable, Hollywood just needs spend more money instead of making it better [11].To some extent, their findings are consistent with those emphasizing the importance of the volume of WOM because higher budget scale generally means higher promotion level and more famous casts which in turn lead to higher WOM volume. Moreover, Basuroy, Chatterjee and Ravid (2003) find that big budgets and stars can serve as an insurance policy. While star power may not be needed if a movie gets good reviews, it can significantly lessen the impact of negative reviews and the same is for budget [3]. Complex as this theoretical network may look, a logical relation between all these different points of view does exist, and we will discuss it and attempt to find a way which ensures all these considerations are carefully included later in this paper.

Meaning \& Purpose. Besides the past research on WOM which we aim to extend, another fact also shed light on the meaning of this paper. The past research is mainly based on the data obtained from western movie market especially America's market. Researchers draw samples from western movies, therefore, the conclusions they reach generally apply to western movie markets. However, with the progress of globalization and development of movie industry in other countries such as China, which comprises the second largest proportion of the total box office revenues all around the world, the effectiveness of the experimental results based on data obtained from western movie markets remains to be confirmed in a different context. As to this, we base our research on China's movie market and examine whether those theories still work in this rapidly-developing market. The movie industry has already achieved the flow of capital all around the world except China. However, the growing number of Sino-foreign co-production indicates the irreversible trend in which Chinese movies are integrating into the world. Furthermore, under the restriction of import quota policy, foreign movies still constantly account for nearly half of the annual total box office generated from China's movie market. As a result, applying those experimental results to China's movie market to examine their effectiveness can lay a firm theoretical foundation for foreign firms to manage their marketing strategies and advertising campaigns in order to make their products successful in China.

The purpose of this paper is to quantify the impact of WOM in the context of China's movie market and find out the factors which determine the trend of box office performance. We choose the ratio of the box office revenue in the first week to the box office revenue in the entire screening cycle of a movie as the proxy variable for the trend of the box office performance and investigate the correlations between the proxy variable and WOM volume and valence. We set the WOM volume in the form of the number of ratings on Douban Movie, the most widely-used online platform full of information and users' ratings related to movies in China. Besides, we set the WOM valence in the form of average user ratings on Douban Movie.

Using the ratio of the box office revenue in the first week to the box office revenue in the entire screening cycle of a movie as the proxy variable can effectively mitigate the effects of different budget levels and popularity of directors and casts as we mention above. It can also reduce effects of bias due to different movies genres. We conduct our experiment under the assumption in which 
the budgets and stars determine the lower limit of the box office revenue whereas WOM determine its future trend. This assumption is underpinned by previous research which suggests budgets and stars do influence the box office performance and WOM exerts a significant impact on the revenue. We presume that the effects of budgets and stars are embodied in the first-week performance when WOM hasn't broadly disseminated and consumers are generally driven by their individual preferences for directors or casts and they are prone to be influenced by propaganda campaigns. However, WOM exerts more power on the trend after the first week. As WOM keeps widely spreading, consumers who are indifferent at the release stage receive more and more information about the movie from mouths of people surrounding them. If the reviews are positive, they may choose to watch it. If the reviews are negative, they may choose not to watch it. Or they may just follow suit as the volume of WOM keep accumulating. All their behaviors are ultimately reflected in the box office performance. By investigating the correlations between the proxy variable for the trend of the box office performance and WOM volume and valence, we can find out which factor really works.

About Douban Movie. Douban Movie is one of the most visited platforms in China and the most authoritative and convincing source of information on movies. Douban Movie allows users to voice their opinions on the quality of movies through rating.

Why do we choose Douban? Generally there are four reasons:

I. Compared to ticket purchasing applications such as Maoyan Movie, Taobao Movie and Weibo Movie, Douban Movie has a large user group with relatively higher professionalism in terms of movie appreciation level. These users are prone to apply a rather strict set of standards to judge a movie. Moreover, most of the users in Douban Movie are labeled as artsy youths (a term used to describe young people who love culture and arts). Because of large quantities of movie viewing experience, these people have a certain level of aesthetic ability with regard to experiential media products such as movies, thus laying a firm foundation for giving an unbiased and relatively professional remark on a particular movie. In contrast, users rating on ticket purchasing applications generally consist of ordinary consumers. Their remarks on movies lack professionalism and prudence compared to those of the users on Douban Movie. Besides, some remarks are diametrically opposed, making it difficult to reach a general consensus on the quality of the movies. In this way, scores on these applications fail to function well without a representative reflection of the overall quality of the movies.

II. Scores on Douban Movies are generally more objective and the general distribution of scores conforms to the traditional $60 \%$-passing system. To put it from another way, the distribution of scores allow users to judge a movie more intuitively basing on whether its score exceeds 6 out of 10.

As an independent rating platform, users need to login to their accounts before they can make remarks on movies. Just before the action of rating, users' wills to remark on movies have already been generated internally which means users are likely to make a remark which is more consistent with its viewing experience in a responsible way. By comparison, users purchase movie tickets on applications such as Maoyan Movie, Taobao Movie and Weibo Movie. After screening, these applications deliver corresponding notifications to users, prompting them to rate movies they have just watched. This passive rating mechanism gives rise to a possibility that some users may simultaneously rate all the movies notified which haven't been rated. The existence of this possibility will dramatically reduce the degree of accuracy and responsibility of rating, making the scores on ticket purchasing applications less objective and representative compared to those on Douban Movie. 
III. Because of Endowment Effect, scores on ticket purchasing applications are higher than they should be. Users on ticket purchasing applications are prompted to rate movies they have just watched by notifications. Under this mechanism, most of the users who rate the movies just complete the consumption- they purchase a movie ticket on the application and watch the movie in cinema. Because they just pay a certain amount of price to obtain that service, according to Endowment Effect, they tend to ascribe more value to the movies they have just watched. In other words, they will probably give a higher-than-normal remark on the real quality of the movies. Moreover, the tendency of ascribing more value to movies results in the distribution of scores on ticket purchasing applications being too dense, making it difficult for users or analysts to differentiate the quality of different movies, not to mention doing further research.

IV. We draw all the movies listed in Top Rated Movies on IMDB and compare the ratings to those of the same movies on Douban Movie. The results show that the ratings of the same movies on both sites have a high degree of similarity. This property of Douban Movie allows the possibility of extending the findings to the global movie market, making the results of experimental study more significative in an international context.

\section{Data and Methodology}

Model and Dependent variable selection. In our study, we use the Linear Regression Model to define the correlations between the proxy variable for the trend of the box office performance (M) and those for WOM volume (V) and valence (Q). Once we have trained a set of feature weights, we could then generate the linear regression equation as follows:

$$
\mathrm{M}=\theta_{0}+\theta_{1} * \mathrm{Q}+\theta_{2} * \mathrm{~V}+\varepsilon_{\mathrm{i}}
$$

Where

$\mathrm{M}=$ the ratio of box office revenue in the first week to that in the entire screening cycle

$\theta_{\mathrm{i}}=$ weights

$\mathrm{Q}=$ the average ratings on Douban Movie

$\mathrm{V}=\ln$ (the number of ratings on Douban Movie)

$\varepsilon_{\mathrm{i}}=$ regression error term

We collect data related to box office of the corresponding movies included in our sample from the Maoyan Pro and figure out the ratio of the box office revenue in the first week to the box office revenue in the entire screening cycle of the movies and set the proxy variable as $M$. Then 1- M represents the rate of survival which means how many proportions of total revenues can be generated after the first week of the screening cycle of a movie. These data are critical to our following study.

We choose average user rating as the proxy variable for WOM valence, and choose the number of ratings as the proxy variable for WOM volume. The reasons for the selection of proxy variable have been explicitly expressed in the last section. We set the proxy variable for WOM valence as Q, and the proxy variable for WOM volume as V.

Sample characteristic \& source. Our data include a random sample of 100 movies released in China between 2013 to 2017. We gather our data from Maoyan Pro, or so-called Maoyan Professional Edition (http://piaofang.maoyan.com). Some past researches focus on major movies. For example, Thorsen, Caroline and Fabian (2013) say that approximately 46 percent of movie ticket sales for major movies are generated in the first week of a new product's release. Nevertheless our study contains a random sample of the movies, whether they are blockbusters or flops. Our 
sample contains 63 Chinese domestic movies and 37 foreign productions. In our sample, $18 \%$ of the movies are released in 2013; 15\%, 2014; 20\%, 2015;21\%, 2016; and 26\%, 2017. This distribution closely matches the distribution of all movies released in China between 2013 and $2017(18.17 \%$, $2013 ; 17.60 \%, 2014 ; 19.53 \%, 2015 ; 21.98 \%, 2016$; and $22.72 \%, 2017)$.

Table 1 below illustrates the characteristics of our sample in terms of the three measurements. As we can see, generally 64.4 percent of movie ticket sales are generated in the first week of a new product's release. Besides, movies included in our sample can score 6.732 points on average. And the average number of people rating each movie is 166347.280.

It should be noted that the number of ratings is rather large in amount and changes by a wider margin compared to the rating itself, thus we nondimensionalize this variable by taking natural logarithms of the number of ratings on Douban Movie.

Table 1 Statistic Summary

\begin{tabular}{lccc}
\hline \multicolumn{1}{c}{ Variable } & Mean & Median & St. Deviation \\
\hline $\begin{array}{l}\text { Ratio of box office revenue } \\
\text { in the first week to that in } \\
\text { the entire screening cycle }\end{array}$ & 0.644 & 0.635 & 0.197 \\
$\begin{array}{l}\text { Average ratings on Douban } \\
\text { Movie }\end{array}$ & 6.732 & 7.350 & 1.640 \\
$\begin{array}{l}\text { Number of ratings on } \\
\text { Douban Movie) }\end{array}$ & 166347.280 & 151333.000 & 122879.528 \\
\hline
\end{tabular}

\section{Results}

Table 2 Regression Result

\begin{tabular}{|c|c|c|c|c|c|}
\hline \multirow[b]{2}{*}{ Variable } & \multicolumn{2}{|c|}{$\begin{array}{l}\text { Unstandardized } \\
\text { Coefficients }\end{array}$} & \multicolumn{2}{|c|}{$\begin{array}{l}\text { Standardized } \\
\text { Coefficients }\end{array}$} & \multirow[b]{2}{*}{$\begin{array}{l}\text { Significance } \\
\text { (p-value) }\end{array}$} \\
\hline & B & Std. Error & Beta & t-Value & \\
\hline Constant & 1.803 & 0.099 & & 18.254 & $<0.001$ \\
\hline $\begin{array}{l}\text { Average ratings on } \\
\text { Douban Movie }\end{array}$ & -0.040 & 0.009 & -0.331 & -4.401 & $<0.001$ \\
\hline $\begin{array}{l}\text { ln (Number of ratings } \\
\text { on Douban Movie) }\end{array}$ & -0.078 & 0.010 & -0.558 & -7.420 & $<0.001$ \\
\hline
\end{tabular}

Note: $\mathrm{R}$ Square $=0.637$, Adjusted R Square $=0.630$

We can generate the linear regression equation as follows from Table 2:

$\mathrm{Y}=1.803-0.040 \mathrm{Q}-0.078 \mathrm{~V}$

Both of the significance of the two independent variables is less than 0.05 respectively, showing that both of the two independent variables- the average user rating which represents WOM valence and the natural logarithm of the number of ratings which represents WOM volume, are prominent in this linear regression equation. As a result, at 0.05 of significance level, we think there exists a linear relationship between the dependent variable- the ratio of box office revenue in the first week to that in the entire screening cycle, and two independent variables- the average ratings on Douban Movie and the natural logarithm of the number of ratings on Douban Movie. Furthermore, according to standardized coefficients, the natural logarithm of the number of ratings on Douban 
Movie has a greater impact on dependent variable than the average ratings on Douban Movie does.

As is shown in Table 2, the coefficient of determination, $\mathrm{R}$ square, is big enough in this linear equation equation, giving rise to a high degree of accuracy for prediction. In other words, we can use this equation to effectively predict the dependent variable.

\section{Conclusion and Discussion}

The purpose of this paper is to quantify the impact of WOM in the context of China's movie market and find out the factors which determine the trend of box office performance. From the experimental results, we can draw a conclusion that there is a strong correlation between the trend of movie box office performance and WOM valence and volume which means both of WOM valence and WOM volume can significantly determine the trend of box office performance in the future, though WOM volume seemingly exerts more influence on the trend. This study is in contrast with previous ones that have found that only one factor, either WOM volume or WOM valence, works in predicting the box office performance.

As for the Endogeneity of our model, we assume that users rate movies objectively based on their experiences which means they will not be influenced by the trend of box office performance. Despite the fact in which Chen, Zhang and Gao (2016) find that there exists the anchoring effect in the field of online word-of-mouth when basing their study on Douban Movie's online rating system, their findings are only restricted to WOM valence itself, not relevant to box office performance [12]. So we will not discuss it in this paper.

Having knowledge of the precise correlations between these three variables can assist directors of movie companies in managing their advertising campaigns and marketing strategies. Ability to accurately predict the box office for a movie will help the cinema line determine the propaganda cost and period of showing the movie to maximize the profits (Parimi and Caragea 2013) [13]. For example, if the movie has a relatively poor quality-in the form of average user rating, then its issuer should limit the scale of pre-release in a bid to curb the spreading of negative reviews. They also should invest more on initial propaganda in order to generate more revenues in the first week when negative WOM hasn't broadly disseminated because these movies usually have a much higher ratio of the box office revenue in the first week to the box office revenue in the entire screening cycle.

However, we should notice the outliers which obviously violate the law in our data sample. Their existence may have something to do with the movie schedule. To put it differently, because the consumer demand constantly occurs, if there is no well-matched competitor, consumers will have no choices but to watch whatever they can watch regardless of WOM. In addition, although as the experimental results, the higher the average ratings on Douban Movie are, the more proportion of box office revenues survive after the first week, the rating bands of different movie genres match different box office revenues accordingly. For instance, if a comedy scores over 7 points on Douban Movie, it is likely to perform strongly on the movie market. However, the threshold to success for dramas may exceeds 8 points. Therefore, in our future studies, we will try to include more considerations and variables in an attempt to explain those outliers as well as define the precise relationship between the ratings divided by movie genres and the corresponding box office performance.

\section{References}

[1]. Hennig-Thurau T, Wiertz C, Feldhaus F. Does Twitter matter? The impact of microblogging word of mouth on consumers' adoption of new movies[J]. Journal of the Academy of Marketing Science, 2015, 43(3):375-394. 
[2]. The Wall Street Journal. "Town \& Country" Publicity Proves an Awkward Act[J]. 2001, B1, B6.

[3]. Basuroy S, Chatterjee S, Ravid S A. How Critical Are Critical Reviews? The Box Office Effects of Film Critics, Star Power, and Budgets[J]. Journal of Marketing, 2003, 67(4):103-117.

[4]. Eliashberg J, Shugan S M. Film critics: Influencers or predictors?[J]. Journal of Marketing, 1997, 61(2):68-78.

[5]. Holbrook M B. Popular Appeal versus Expert Judgments of Motion Pictures[J]. Journal of Consumer Research, 1999, 26(2):144-155.

[6]. West P M, Broniarczyk S M. Integrating Multiple Opinions: The Role of Aspiration Level on Consumer Response to Critic Consensus[J]. Journal of Consumer Research, 1998, 25(1):38-51.

[7]. Liu Y. Word of Mouth for Movies: Its Dynamics and Impact on Box Office Revenue[J]. Journal of Marketing, 2006, 70(3):74-89.

[8]. Duan W, Gu B, Whinston A B. The dynamics of online word-of-mouth and product sales-An empirical investigation of the movie industry[J]. Journal of Retailing, 2008, 84(2):233-242.

[9]. Chevalier J A, Mayzlin D. The Effect of Word of Mouth on Sales: Online Book Reviews[J]. Social Science Electronic Publishing, 2006, 43(3):345-354.

[10]. Chintagunta P K, Gopinath S, Venkataraman S. The Effects of Online User Reviews on Movie Box-Office Performance: Accounting for Sequential Rollout and Aggregation Across Local Markets[J]. Marketing Science, 2010, 29(5):944-957.

[11]. Wasserman M, Mukherjee S, Scott K, et al. Correlations between user voting data, budget, and box office for films in the internet movie database[J]. Journal of the Association for Information Science \& Technology, 2015, 66(4):858-868.

[12]. Chen Subai, Zhang Yicheng, Gao Shijie. An empirical study of on anchoring effect in the field of online word-of-mouth: Take Douban movie's online rating system as an example[J]. Journal of International Communication, 2016(3):34-48.

[13]. Parimi R, Caragea D. Pre-release box-office success prediction for motion pictures[C]// International Workshop on Machine Learning and Data Mining in Pattern Recognition. Springer, Berlin, Heidelberg, 2013:571-585. 